\title{
High-energy in-beam neutron measurements of metal-based shielding for accelerator-driven spallation neutron sources
}

\author{
D. D. DiJulio, ${ }^{1,2,}$ C. P. Cooper-Jensen, ${ }^{1,3}$ H. Björgvinsdóttir, ${ }^{1,3}$ Z. Kokai, ${ }^{1}$ and P. M. Bentley ${ }^{1,3}$ \\ ${ }^{1}$ European Spallation Source ERIC, P.O. Box 176, SE-221 00 Lund, Sweden \\ ${ }^{2}$ Division of Nuclear Physics, Lund University, SE-221 00 Lund, Sweden \\ ${ }^{3}$ Department of Physics and Astronomy, Uppsala University, Uppsala SE-751 20, Sweden
}

(Received 17 December 2015; published 31 May 2016)

\begin{abstract}
Metal-based shielding plays an important role in the attenuation of harmful and unwanted radiation at an accelerator-driven spallation neutron source. At the European Spallation Source, currently under construction in Lund, Sweden, metal-based materials are planned to be used extensively as neutron guide substrates in addition to other shielding structures around neutron guides. The usage of metal-based materials in the vicinity of neutron guides however requires careful consideration in order to minimize potential background effects in a neutron instrument at the facility. Therefore, we have carried out a combined study involving high-energy neutron measurements and Monte Carlo simulations of metal-based shielding, both to validate the simulation methodology and also to investigate the benefits and drawbacks of different metal-based solutions. The measurements were carried out at The Svedberg Laboratory in Uppsala, Sweden, using a $174.1 \mathrm{MeV}$ neutron beam and various thicknesses of aluminum-, iron-, and copper-based shielding blocks. The results were compared to Geant 4 simulations and revealed excellent agreement. Our combined study highlights the particular situations where one type of metal-based solution may be preferred over another.
\end{abstract}

DOI: 10.1103/PhysRevAccelBeams.19.053501

\section{INTRODUCTION}

At an accelerator-driven spallation source, metal-based shielding plays an important role in the attenuation of highenergy neutrons. These neutrons, which are generated after the impact of the proton beam with the target, can have high energies which reach up to the primary beam energy $(\mathrm{GeV})$ $[1,2]$ and can induce further secondary neutrons deep in the shielding around the target region. This situation leads to massive biological shielding requirements, compared to those at reactor-based sources, where fission processes only lead to secondary neutrons up to $20 \mathrm{MeV}$ [2]. Typically, the design of a biological shield at a spallation source can consist of several meters of iron and is additionally followed by a concrete layer [2,3].

Not only is metal-based shielding important for radiation safety purposes at spallation neutron sources, it also can serve an additional role in minimizing background radiation at the sample position of a neutron scattering instrument installed at the facility. The usable signal for a neutron instrument contains low-energy neutrons on the order of $\sim \mathrm{meV}$ which are guided from moderators placed near the spallation target to the sample position some tens of meters

\footnotetext{
*douglas.dijulio@esss.se

Published by the American Physical Society under the terms of the Creative Commons Attribution 3.0 License. Further distribution of this work must maintain attribution to the author $(s)$ and the published article's title, journal citation, and DOI.
}

away via neutron guides. However, the high-energy neutrons produced during the spallation process in the target can induce secondary neutrons in the shielding of the facility which ultimately reach the sample position of an instrument. This effect can contribute to the appearance of a prompt-pulse background, which has been observed on a number of modern neutron scattering instruments [4,5] and is of great interest to neutron spectroscopy instruments, which may measure during the time period when the proton beam is on. Metal-based shielding, containing iron for example, is key for attenuation of the high-energy neutrons, due to the atomic mass and density, which leads to an increased attenuation at higher energies [6], compared to standard concrete.

The usage of metal-based shielding near neutron guides however requires careful consideration in order to minimize potential background effects in a neutron instrument. In particular, at the European Spallation Source (ESS) [7], currently under construction in Lund, Sweden, it is anticipated that all neutron guides within the common shielding of the facility, which extends to $15 \mathrm{~m}$ for short instruments and $28 \mathrm{~m}$ for long instruments, will use metal-based substrates for the neutron guides due to the conditions imposed by the radiation environment. Metal-based substrates exhibit higher stability against irradiation, mechanical stress, and temperature compared to the traditionally used glass substrates [8]. Of particular interest are aluminum, copper, and steel substrates. On the other hand, metal-based materials can scatter neutrons 
back into the guide. For a short instrument at the facility, such as LOKI [9], which has a source-to-detector distance of $30 \mathrm{~m}$, a significant fraction of the guide substrates of the instrument will be made from metal. This means that proper selection of the metal-based shielding is critical for reducing the albedo scattering within the neutron guide itself in order to limit the background at the sample position. A key aspect of the background mitigation strategy at ESS is thus to use the most appropriate and effective materials for radiation attenuation at selected locations along a neutron beam line, which could both be in the form of substrates and/or collimator blocks, for example $[4,5,10]$.

For the above-mentioned reasons, accurate simulation of neutron beam lines during the instrument engineering design phase is therefore crucial for achieving low instrument backgrounds. A key question is then: How well do modern simulation packages reproduce measured data at energies relevant to spallation neutron sources? Of particular relevance for the current study is the suitability of Geant4 [11] for simulating neutron beam lines, as it has seen little application within this field previously. The usage of Geant 4 for these types of calculations has increased in recent years and at the ESS it is one of the standard simulation packages employed for modeling shielding and detector components [12-15].

In the past, a variety of measurements have been carried out for benchmarking purposes [15-20]. These measurements however have largely focused on iron/concrete shields or more complex shielding scenarios with the incident neutron energies ranging over the entire spallation source energy spectrum. Measurements with alternative materials at monoenergetic energies can be seen as complementary to these previous studies and can provide more precise benchmarks for simulation packages. It can also be mentioned that new shielding data can be useful for other applications of proton accelerators, such as those at hospital based facilities [21-23].

In the current work, we have carried out a combined measurement and simulation study of metal-based shielding for accelerator-driven spallation neutron sources. We focus on the neutronic properties of the shielding. In the following, we present: (1) a benchmark comparison between measurements and simulations of metal-based shielding at energies relevant to spallation neutron source environments; (2) an analysis of the scattered neutrons from metal-based shielding for investigating the potential background reduction benefits; and lastly discuss the potential advantages of steel, copper, and aluminum shielding and present some situations where one type of metal may be preferred over another.

\section{IN-BEAM MEASUREMENTS}

The measurements were carried out at The Svedberg Laboratory (TSL) [24] in Uppsala, Sweden, using the

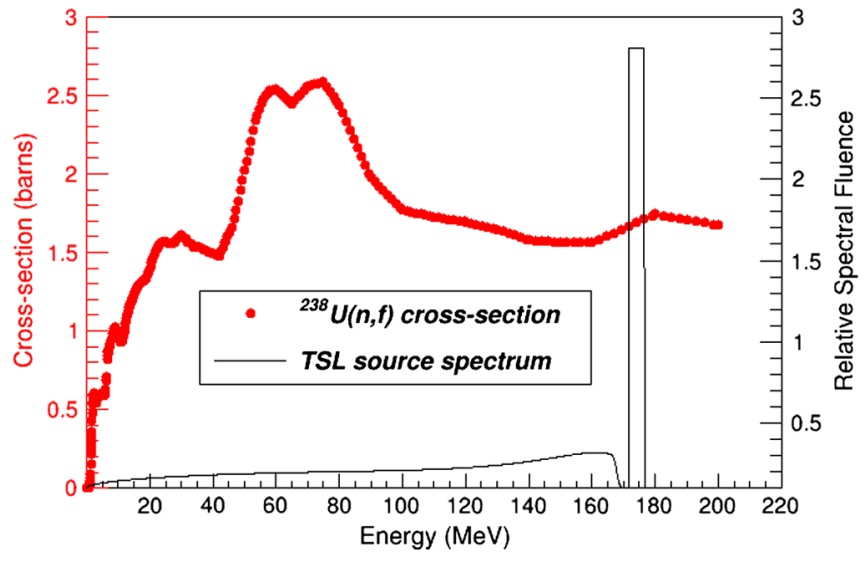

FIG. 1. The incident neutron spectrum used in the TSL measurements, calculated using the algorithm in [26], and the ${ }^{238} \mathrm{U}(n, f)$ cross section [27], which highlights the sensitive range of the neutron fluence monitor used in the measurements.

Quasi-Monoenergetic Neutron (QMN) [25] facility. Neutrons with an average energy of $174.1 \mathrm{MeV}$ were produced with a proton beam energy of $178.5 \mathrm{MeV}$ incident on a $23.5 \mathrm{~mm}$ thick $99.99 \%$ pure ${ }^{7} \mathrm{Li}$ target with a size of $20 \times 32 \mathrm{~mm}^{2}$. The beam spot size on the target was $20 \mathrm{~mm}$ in diameter. A cylindrical collimator, with a diameter of $75 \mathrm{~mm}$, was used to shape the beam of neutrons which was incident on a series of sample blocks at $3.73 \mathrm{~m}$ from the target position. The incident neutron energy spectrum is given in Fig. 1. The spectrum has been calculated using the algorithm from Ref. [26].

A neutron fluence monitor was placed at a distance of $4.29 \mathrm{~m}$ from the target position to detect the transmitted neutrons. The neutron fluence monitor was a fission fragment ionization chamber based on the ${ }^{238} \mathrm{U}(n, f)$ cross section, which is shown in Fig. 1 and taken from the TENDL-2014 library [27]. The diameter of the fissile targets was $7 \mathrm{~cm}$. Events recorded in the monitor were written to a log file for further analysis. In the open-beam case, approximately $60 \%$ of the counts recorded in the monitor arise from the neutrons in the continuum below $170 \mathrm{MeV}$ and approximately $40 \%$ from the pseudomonoenergetic line at $174 \mathrm{MeV}$.

Three different materials were investigated during the measurements. These included blocks of aluminum alloy 6082-T6, steel CK45 (medium carbon steel), and a high conductivity phosphorous copper $\mathrm{Cu}-\mathrm{HCP}$. Each block was $10 \mathrm{~cm}$ thick and $20 \mathrm{~cm}$ in width and height. Four blocks of each material were studied, amounting to a total maximum thickness of $40 \mathrm{~cm}$ in all cases examined. The material compositions of the shielding blocks are shown in Table I. Since these materials are industrial standard material we do not know the exact chemical composition. However, we have found through simulations that the small variations in the chemical compositions change the simulated results by less than $0.5 \%$. 
TABLE I. Material composition of the shielding blocks used in the in-beam study. Elemental compositions are given in weight percents.

\begin{tabular}{lccc}
\hline \hline Element & AL6082-T6 & Cu-HCP & Steel CK45 \\
\hline $\mathrm{Al}$ & $95.2-98.3$ & & \\
$\mathrm{Cr}$ & $\leq 0.25$ & & $\leq 0.40$ \\
$\mathrm{Cu}$ & $\leq 0.10$ & $\geq 99.95$ & \\
$\mathrm{Fe}$ & $\leq 0.50$ & & 97.86 \\
$\mathrm{Mg}$ & $0.60-1.20$ & & \\
$\mathrm{Mn}$ & $0.40-1.0$ & & 0.65 \\
$\mathrm{Si}$ & $0.70-1.30$ & & $\leq 0.40$ \\
$\mathrm{Ti}$ & $\leq 0.10$ & & \\
$\mathrm{Zn}$ & $\leq 0.20$ & & \\
$\mathrm{Ag}$ & & $\leq 0.015$ & \\
$\mathrm{P}$ & & $0.002-0.007$ & \\
$\mathrm{Bi}$ & & $\leq 0.0005$ & \\
$\mathrm{~Pb}$ & & & \\
$\mathrm{C}$ & & & $\leq .005$ \\
$\mathrm{Mo}$ & & & $\leq 0.46$ \\
$\mathrm{Ni}$ & & & 7.84 \\
$\mathrm{Cr}+\mathrm{Mo}+\mathrm{Ni}$ & & 8.94 & \\
$\rho\left(\frac{\mathrm{g}}{\mathrm{cm}^{3}}\right)$ & 2.7 & & \\
\hline \hline
\end{tabular}

\section{MONTE CARLO SIMULATIONS}

The experimental setup was modeled using the Monte Carlo simulation package Geant4 version 10.0 [11]. The simulations included the geometrical setup described above, the properties of the incoming neutron beam, and the material properties for the shielding blocks. In the simulations, the divergence of the neutron beam was approximated to be $\sim 0.8^{\circ}$ originating at the location of the neutron production target. This resulted in a beam spot diameter of $8.6 \mathrm{~cm}$ on the front face of the first shielding block. The number of neutrons leaving the surfaces of the shielding blocks and at the position of the monitor for a single simulation was stored in a log file. This latter information was folded with the ${ }^{238} \mathrm{U}(n, f)$ cross section, shown in Fig. 1, for direct comparison to the measurements.

Two physics lists were used in the simulations and included the QGSP_INCLXX_HP and QGSP_BERT_HP lists [28], which are generally recommended for shielding applications [29]. The abbreviation QGS stands for the quark-gluon string model, P for precompound, INCLXX for the Liège Intranuclear Cascade model [30,31] and BERT for the Bertini cascade model $[32,33]$. HP stands for the high-precision neutron package [28], which includes evaluated neutron data, called G4NDL4.4, for neutron interactions below $20 \mathrm{MeV}$. The data largely comes from the ENDF/B-VII.0 libraries. The Geant4 model was implemented in the simulation framework provided by the ESS Detector Group [12], which provides tools and developments for neutron detection and shielding calculations.

\section{RESULTS}

\section{A. Benchmarking the simulations}

The measured results for varying thicknesses of shielding material are compared to the Geant4 simulations in Fig. 2. The quantity $R / R_{0}$ represents the counts in the fluence monitor as measured and simulated for the indicated thickness of a shielding block divided by the counts without any shielding block in the beam. It can be seen that the simulations using both models reproduce excellently the trends of the measurements for all three materials investigated. The copper- and iron-based materials have nearly identical performance and as expected provide a higher attenuation of the beam compared to the aluminumbased material. These results highlight the suitability of Geant4 for simulating neutron interactions at high energies and in these types of materials.

An interesting observation from the data shown in Fig. 2 is that three blocks of aluminum-based material results in a similar measured performance as one block of either of the two other materials. To further explore this, we also investigated the properties of the scattered neutrons at the position of the neutron monitor for these combinations. For example, the simulated energies of the particles after a single copper- or iron-based block compared to three aluminum-based blocks are shown in Fig. 3. The energy spectrum after the aluminum-based blocks is indeed similar in shape and magnitude to the copper- or iron-based block cases, substantiating the above-mentioned observation.

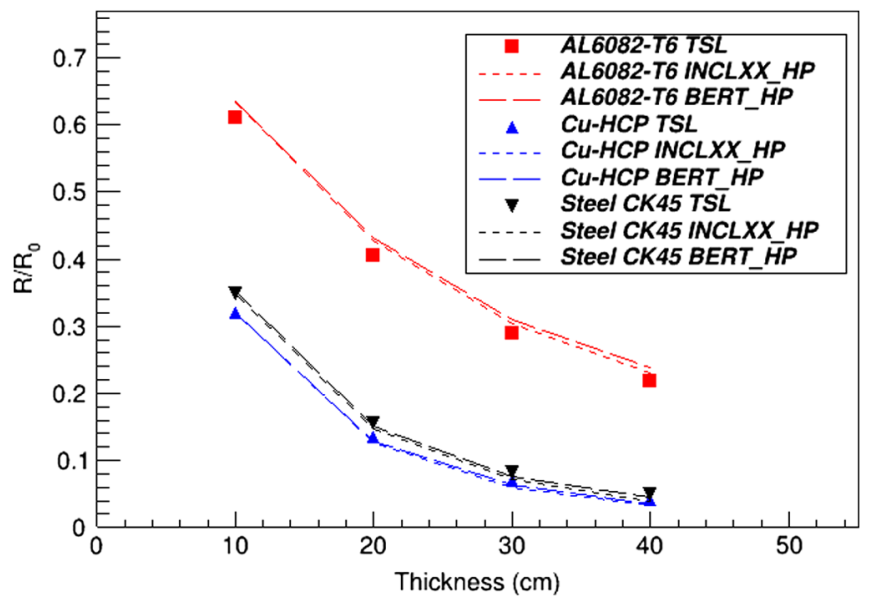

FIG. 2. Comparison of the Geant 4 simulations and TSL measurements for varying thicknesses of shielding materials. The points represent the measured data and the error bars are smaller than the markers. The simulations were carried out for the same thicknesses and the lines are drawn to guide the eye. The statistical error for the simulated data is less than $0.2 \%$. The quantity $R / R_{0}$ represents the counts in the fluence monitor as measured and simulated for the indicated thickness of a shielding block divided by the counts without any shielding block in the beam. 


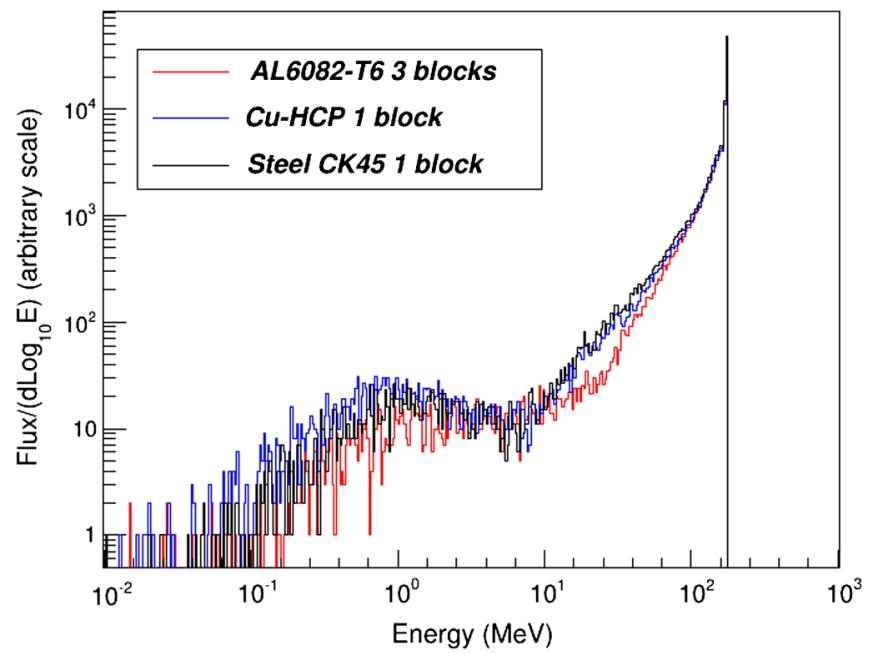

FIG. 3. Comparison of the Geant4 simulated neutron energy spectra at the monitor position for $\mathrm{Cu}-\mathrm{HCP}(10 \mathrm{~cm})$, steel CK45 $(10 \mathrm{~cm})$ and AL6082-T6 $(30 \mathrm{~cm})$. The simulations used the QGSP_INCLXX_HP physics list.

We also investigated the effect of laminated shields compared to pure shielding with the TSL primary beam and the results for some selected combinations are given in Table II. In both the two block and four block cases, we found that the best performance was provided by the pure copper-based material combinations, followed by the mixed iron- and copper-based combinations, and then the pure iron-based combinations. This result can be understood from the simulated energy spectra for the two block case and is presented in Fig. 4. The figure shows a comparison of the neutron energy spectra at the position of the monitor for the $\mathrm{Fe}-\mathrm{Cu}$ and $\mathrm{Cu}-\mathrm{Fe}$ configurations. The observed scattering into the resonance regions below $1 \mathrm{MeV}$ only contributes to a small fraction of the transmitted beam. At the primary beam energy, the attenuation is driven by the atomic mass and density and therefore the copper-based material yields the best performance. However, it can be pointed out that neutrons transmitted through the window around $29 \mathrm{keV}$ in $\mathrm{Fe}$ is visible in the

TABLE II. Comparison of measurements and simulations for different combinations of shielding blocks. Fe refers to steel $\mathrm{CK} 45$ and $\mathrm{Cu}$ to $\mathrm{Cu}-\mathrm{HCP}$. The values in the table correspond to the quantity $R / R_{0}$, as described in the text.

\begin{tabular}{lccc}
\hline \hline Combination & Measurement & INCLXX_HP & BERT_HP \\
\hline $\mathrm{Cu} \mathrm{Cu}$ & $0.1338(12)$ & $0.1260(6)$ & $0.1289(6)$ \\
$\mathrm{Cu} \mathrm{Fe}$ & $0.1439(12)$ & $0.1350(2)$ & $0.1393(2)$ \\
$\mathrm{Fe} \mathrm{Fe}$ & $0.1561(12)$ & $0.1461(7)$ & $0.1509(7)$ \\
$\mathrm{Fe} \mathrm{Cu}$ & $0.1436(12)$ & $0.1369(2)$ & $0.1408(2)$ \\
$\mathrm{Cu} \mathrm{Cu} \mathrm{Cu} \mathrm{Cu}$ & $0.0408(4)$ & $0.0317(3)$ & $0.0354(3)$ \\
$\mathrm{Cu} \mathrm{Fe} \mathrm{Cu} \mathrm{Fe}$ & $0.0450(4)$ & $0.0347(1)$ & $0.0390(1)$ \\
$\mathrm{Fe} \mathrm{Fe} \mathrm{Fe} \mathrm{Fe}$ & $0.0498(4)$ & $0.0387(3)$ & $0.0443(3)$ \\
\hline \hline
\end{tabular}

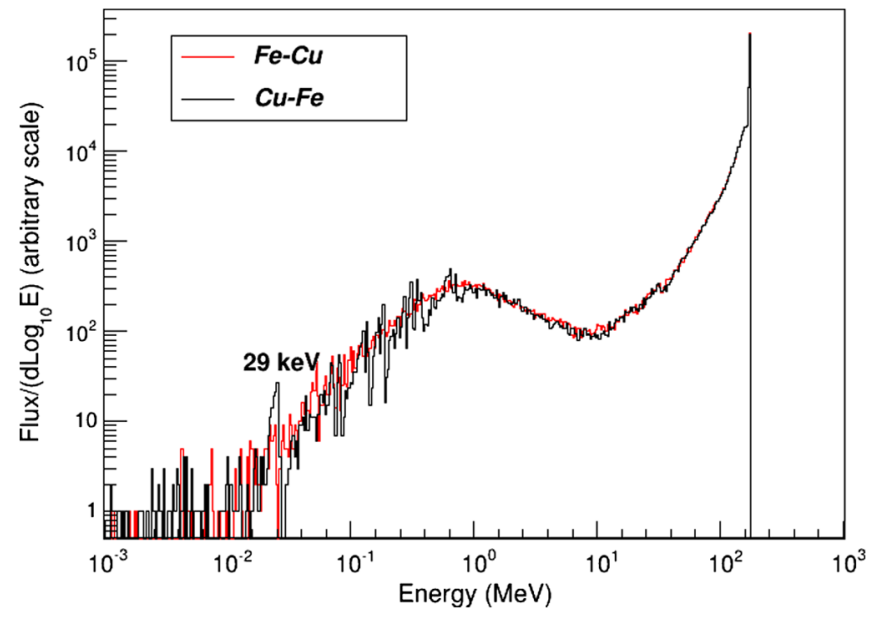

FIG. 4. Comparison of the Geant4 simulated $\mathrm{Fe}-\mathrm{Cu}$ and $\mathrm{Cu}-\mathrm{Fe}$ energy spectra as used in the TSL measurements. Fe refers to steel CK45 and $\mathrm{Cu}$ to $\mathrm{Cu}-\mathrm{HCP}$. The simulations used the QGSP_INCLXX_HP physics list.

$\mathrm{Cu}-\mathrm{Fe}$ configuration, while it is not visible in the $\mathrm{Fe}-\mathrm{Cu}$ configuration.

Lastly, it can be mentioned that generally the BERT_HP model yielded better agreement with the measurements compared to the INCLXX_HP model. The differences between the measurements and simulations in Table II reach a maximum of $30 \%$ for the INCLXX_HP model and $15 \%$ for the BERT_HP model, while the differences between the two models is maximum $14 \%$. However, it should also be mentioned that for the four block aluminum case in Fig. 2, the INCLXX_HP model performed slightly better, with a $5 \%$ difference compared to $8 \%$ for the BERT_HP model.

\section{B. Analysis of the scattered particles}

In order to understand the potential scenarios where one type of metal may be preferred over another along a neutron beam guide, we carried out an analysis of the scattered particles leaving the shielding blocks. For the comparison, we used the observation from the data presented in Fig. 2 which shows that three blocks of aluminum-based material results in a similar performance as one block of either of the two other materials. The simulated results of the number of scattered neutrons for each block scenario from the different sides of a block are shown in Table III. The front of a block refers to the face closest to the detector position while the back refers to the face where the beam is incident on a block. The simulations were carried out for the TSL block geometries and also blocks which were infinite in size in the directions perpendicular to the incident beam. The results are normalized to the number of incident neutrons. The average energies of these neutrons are also given in Table IV.

In the infinite block case, all neutrons are scattered either in the forward or backward directions. The copper-based 
TABLE III. Simulated scattered number of neutrons out of the surfaces of the single block and three block scenarios described in the text. The numbers are given normalized to the number of incident neutrons. B refers to block. The results are from the Geant4 simulations using the QGSP_INCLXX_HP physics list.

\begin{tabular}{lcccc}
\hline \hline & Front & Side & Back & Total \\
\hline TSL size blocks & & & & \\
Cu-HCP (1 B) & 1.12 & 0.42 & 0.42 & 1.96 \\
Steel CK45 (1 B) & 1.04 & 0.37 & 0.32 & 1.73 \\
AL6082-T6 (3 B) & 0.64 & 0.72 & 0.10 & 1.46 \\
Infinite X-y blocks & & & & \\
Cu-HCP (1 B) & 1.35 & 0.00 & 0.62 & 1.97 \\
Steel CK45 (1 B) & 1.25 & 0.00 & 0.50 & 1.74 \\
AL6082-T6 (3 B) & 1.07 & 0.00 & 0.40 & 1.47 \\
\hline \hline
\end{tabular}

block scatters the most neutrons in the forward directions, followed by the iron-based and aluminum-based blocks. The same trend can be seen in the backward directions. Furthermore, in total more neutrons are emitted from the block(s) than are incident on the block(s) for all three cases. It is also interesting to note the high secondary production for the single copper- and iron-based blocks, compared to the three aluminum-based blocks. For the average energies, the neutrons emanating in the forward directions for the copper-based block have the lowest energies, while the neutrons from the aluminum-based blocks have the highest energies. Neutrons emanating in the backward directions have similar energies in all three cases.

As the blocks get smaller in the transverse dimension, the number of neutrons coming out of the front and back faces decreases and the number of neutrons emanating from the side surfaces of the blocks increases. Similar to the infinite block case, the copper-based block scatters the most neutrons in the forward directions and the aluminum-based blocks the least. The aluminum-based configuration however scatters the most neutrons out of the side surfaces (0.72) compared to the other two blocks ( 0.42 and 0.37 for $\mathrm{Cu}-\mathrm{HCP}$ and steel CK45). While this is true, the side surface of the aluminum blocks is 3 times larger. This leads

TABLE IV. Simulated average energy $(\mathrm{MeV})$ of the neutrons out of the surfaces of the single block and three block scenarios described in the text. B refers to block. The results are from the Geant4 simulations using the QGSP_INCLXX_HP physics list.

\begin{tabular}{lcccc}
\hline \hline & Front & Side & Back & Total \\
\hline TSL size blocks & & & & \\
Cu-HCP (1 B) & 74 & 7 & 3 & 44 \\
Steel CK45 (1 B) & 82 & 8 & 3 & 52 \\
AL6082-T6 (3 B) & 110 & 19 & 4 & 58 \\
Infinite X-y blocks & & & & \\
Cu-HCP (1 B) & 62 & 0 & 2 & 44 \\
Steel CK45 (1 B) & 69 & 0 & 3 & 50 \\
AL6082-T6 (3 B) & 73 & 0 & 3 & 54 \\
\hline \hline
\end{tabular}

to the surface density of the neutrons on the side surfaces being about a factor of 2 times lower than the side surface density of the iron- and copper-based blocks. The average energy of the neutrons emanating from the front and side surfaces is lowest for the copper-based block and highest for the aluminum-based blocks. The average energy of the backscattered neutrons is similar in all three cases. The above-mentioned findings will be discussed in more detail in the following section.

\section{DISCUSSION}

The results presented in the previous sections illustrate the neutronic properties of metal-based materials for shielding applications at accelerator-driven spallation neutron sources. For example, Fig. 2 shows that three blocks of aluminum-based shielding material provide roughly the same performance for the measurements as one block of copper- or iron-based materials. However, each neutron removed from the primary beam will generate additional secondaries within the block which can undergo further collisions and lose energy before escaping the shielding material thickness. These effects are illustrated in Tables III and IV. It can be seen that copper-based materials, and then iron-based materials, produce the largest number of scattered neutrons leaving a block. The average energy of the neutrons leaving the blocks is also lower in energy than in the aluminum-based block case. In applications where a high-energy primary beam needs to be slowed down efficiently and in a limited physical space, iron- and copper-based materials offer the best performance. The aluminum-based material, on the other hand, produces less forward going scattered neutrons and also emits less scattered neutrons out of the side faces and back face per $\mathrm{cm}^{2}$. The energies of these neutrons are however on average higher than in the copper- and iron-based block cases. Depending on the shielding requirements, and particularly if limited space is not an issue, aluminumbased materials may however be an alternative which could be attractive.

The above results support the conclusion that aluminumbased materials could be the preferable option as a metal guide substrate or shielding in the immediate vicinity of a neutron guide, especially at locations of an instrument close to the sample position. At locations closer to the spallation target, one could however envision using copper or steel substrates, as they provide better attenuation of the primary beam and due to the longer distance from the sample position there is a lower probability for the scattered neutrons to reach the end of an instrument. Careful analysis of the energies of the emitted neutrons and their locations relative to the instrument detector positions should therefore be considered in the shielding material selection process as each material has benefits and disadvantages.

In addition to the above, aluminum has a lower cross section for neutron production via photon absorption in the 
giant-dipole resonance (GDR) compared to iron and copper [34]. High-energy photons, which cover the energy range of GDR, are also produced during the spallation process in the target and enter the neutron guides. The usage of aluminum-based materials would minimize neutron production through this mechanism in the vicinity of neutron guides. An additional study on this topic could thus be considered of great interest.

Lastly, it should be mentioned that the choice of which metal-based material to use in selected locations along a beam line depends on a number of other factors, which include for example cost, weight limitations, and activation properties, to name a few. Furthermore, the results of the calculations shown in this work were carried out for specific beam combinations and shielding block sizes. In realistic applications, as much detail should be included as possible.

\section{CONCLUSIONS}

In summary, we performed a combined measurement and simulation study of the transmission properties of metal-based shielding for accelerator-driven spallation neutron sources. The measurements were carried out at TSL using a $174.1 \mathrm{MeV}$ neutron beam incident on three different metal-based materials. The results were compared to Monte Carlo simulations using Geant 4 and showed good agreement. Our results revealed several potential benefits of different metal-based shielding materials which may also be considered during the shielding material selection process in order to reduce backgrounds on neutron scattering instruments.

\section{ACKNOWLEDGMENTS}

The authors would like to thank Elke Passoth, Alexander Prokofiev, and the staff at TSL for help during various stages of the measurement planning, execution, and analysis of the data presented in this work.

[1] G. J. Russell, H. Robinson, G. L. Legate, and R. Woods, in Proceedings of the ICANS-X Conference, Los Alamos, New Mexico, 1988, http://www.neutron.anl.gov/ipns/icans .html.

[2] G. S. Bauer, Physics and technology of spallation neutron sources, Nucl. Instrum. Methods Phys. Res., Sect. A 463, 505 (2001).

[3] I. Koprivnikar and E. Schachinger, The biological shield of a high-intensity spallation source: A Monte Carlo design study, Nucl. Instrum. Methods Phys. Res., Sect. A 487, 571 (2002).

[4] N. Cherkashyna et al., High energy particle background at neutron spallation sources and possible solutions, J. Phys. Conf. Ser. 528, 012013 (2014).

[5] N. Cherkashyna et al., Overcoming high energy backgrounds at pulsed spallation sources, arXiv:1501.02364.
[6] A. H. Sullivan, A Guide to Radiation and Radioactivity Levels Near High Energy Particle Accelerators (Nuclear Technology Publishing, Ashford, UK, 1992), Section 1.1.5, ISBN 1870965183.

[7] S. Peggs et al., ESS Technical Design Report No. ESS2013-001 (European Spallation Source, ESS AB, Lund, Sweden).

[8] C. Schanzer, P. Böni, and M. Schneider, High performance supermirrors on metallic substrates, J. Phys. Conf. Ser. 251, 012082 (2010).

[9] A. J. Jackson and K. Kanaki, ESS construction proposal LoKI-A broadband SANS instrument, available online at https://europeanspallationsource.se/.

[10] C. Zendler, D. Martin Rodriguez, and P. M. Bentley, Generic guide concepts for the European Spallation Source, Nucl. Instrum. Methods Phys. Res., Sect. A 803, 89 (2015).

[11] S. Agostinelli et al., Geant4-a simulation toolkit, Nucl. Instrum. Methods Phys. Res., Sect. A 506, 250 (2003).

[12] T. Kittelmann, I. Stefanescu, K. Kanaki, M. Boin, R. Hall-Wilton, and K. Zeitelhack, Geant4 based simulations for novel neutron detector development, J. Phys. Conf. Ser. 513, 022017 (2014).

[13] T. Kittelmann and M. Boin, Polycrystalline neutron scattering for Geant4: NXSG4, Comput. Phys. Commun. 189, 114 (2015).

[14] J. Stenander and D. D. DiJulio, Application of automated weight windows to spallation neutron source shielding calculations using Geant4, Nucl. Instrum. Methods Phys. Res., Sect. A 798, 167 (2015).

[15] N. Cherkashyna, D. D. DiJulio, T. Panzner, E. Rantsiou, U. Filges, G. Ehlers, and P. M. Bentley, Benchmarking shielding simulations for an accelerator-driven spallation neutron source, Phys. Rev. ST Accel. Beams 18, 083501 (2015).

[16] J. V. Siebers et al., Measurement of neutron dose equivalent and penetration in concrete for $230 \mathrm{MeV}$ proton bombardment of $\mathrm{Al}, \mathrm{Fe}$, and $\mathrm{Pb}$ targets, Radiat. Prot. Dosim. 44, 247 (1992).

[17] A. Mazal et al., Shielding measurements for a proton therapy beam of $200 \mathrm{MeV}$ : Preliminary results, Radiation Protection Dosimetry 70, 429 (1997).

[18] T. Nunomiya et al., Measurement of deep penetration of neutrons produced by $800-\mathrm{MeV}$ proton beam through concrete and iron at ISIS, Nucl. Instrum. Methods Phys. Res., Sect. B 179, 89 (2001).

[19] M. Sasaki, N. Nakao, T. Nunomiya, T. Nakamura, A. Fukumura, and M. Takada, Measurements of high energy neutrons penetrated through iron shields using the SelfTOF detector and an NE213 organic liquid scintillator, Nucl. Instrum. Methods Phys. Res., Sect. B 196, 113 (2002).

[20] M. Fragopoulou and M. Zamani, Phenomenological calculations of shielding spallation neutron sources, Nucl. Instrum. Methods Phys. Res., Sect. A 714, 24 (2013).

[21] S. Agosteo, M. Magistris, A. Mereghetti, M. Silari, and Z. Zajacova, Shielding data for 100-250 MeV proton accelerators: Double differential neutron distributions and attenuation in concrete, Nucl. Instrum. Methods Phys. Res., Sect. B 265, 581 (2007). 
[22] S. Agosteo, M. Magistris, A. Mereghetti, M. Silari, and Z. Zajacova, Shielding data for 100-250 MeV proton accelerators: Attenuation of secondary radiation in thick iron and concrete/iron shields, Nucl. Instrum. Methods Phys. Res., Sect. B 266, 3406 (2008).

[23] R.-J. Sheu, Y.-Fu Chen, U.-Tyng Lin, and S.-H. Jiang, Deep-penetration calculations in concrete and iron for shielding of proton therapy accelerators, Nucl. Instrum. Methods Phys. Res., Sect. B 280, 10 (2012).

[24] The Svedberg Laboratory, http://www.tsl.uu.se/.

[25] A. V. Prokofiev, J. Blomgren, O. Byström, C. Ekström, S. Pomp, U. Tippawan, V. Ziemann, and M. Österlund, The TSL neutron beam facility, Radiation Protection Dosimetry 126, 18 (2007).

[26] A. Prokofiev et al., Development and validation of the ${ }^{7} \mathrm{Li}(p, n)$ nuclear data library and its application in monitoring of intermediate energy neutrons, J. Nucl. Sci. Technol. 39, 112 (2002).

[27] TALYS: TENDL-2014, http://www.talys.eu/tendl-2014/; retrieved from https://www-nds.iaea.org/exfor/endf.htm.

[28] GEANT4: User documentation; http://geant4.cern.ch/ support/userdocuments.shtml.
[29] Geant4: Reference physics lists: http://geant4.cern.ch/ support/proc_mod_catalog/physics_lists/useCases.shtml.

[30] A. Boudard, J. Cugnon, J.-C. David, S. Leray, and D. Mancusi, New potentialities of the Liège intranuclear cascade model for reactions induced by nucleons and light charged particles, Phys. Rev. C 87, 014606 (2013).

[31] D. Mancusi, A. Boudard, J. Cugnon, J.-C. David, P. Kaitaniemi, and S. Leray, Extension of the Liège intranuclear-cascade model to reactions induced by light nuclei, Phys. Rev. C 90, 054602 (2014).

[32] H. W. Bertini, Low-Energy intranuclear cascade calculation, Phys. Rev. 131, 1801 (1963).

[33] H. W. Bertini, Intranuclear-Cascade calculation of the secondary nucleon spectra from nucleon-nucleus interactions in the energy range 340 to $2900 \mathrm{MeV}$ and comparisons with experiment, Phys. Rev. 188, 1711 (1969).

[34] International Atomic Energy Agency, Handbook on photonuclear data for applications cross sections and spectra, Report No. IAEA-TECDOC-1178, 2000. 